\title{
The gluino-glue particle and finite size effects in supersymmetric Yang-Mills theory
}

\author{
G. Bergner, ${ }^{a}$ T. Berheide, ${ }^{a}$ G. Münster, ${ }^{a}$ U.D. Özugurel, ${ }^{a}$ D. Sandbrink ${ }^{a}$ \\ and I. Montvay ${ }^{b}$ \\ ${ }^{a}$ Universität Münster, Institut für Theoretische Physik, \\ Wilhelm-Klemm-Str. 9, D-48149 Münster, Germany \\ ${ }^{b}$ Deutsches Elektronen-Synchrotron DESY, \\ Notkestr. 85, D-22603 Hamburg, Germany \\ E-mail: g.bergner@uni-muenster.de, tberheide@gmx.de, \\ munsteg@uni-muenster.de, oezugurel@uni-muenster.de, \\ dirk.sandbrink@uni-muenster.de, montvay@mail.desy.de
}

ABSTRACT: The spectrum of particles in supersymmetric Yang-Mills theory is expected to contain a spin $1 / 2$ bound state of gluons and gluinos, the gluino-glue particle. We study the mass of this particle in softly broken supersymmetric Yang-Mills theory on a lattice by means of numerical simulations. The main focus is the estimation of finite size effects. We extrapolate the mass first to the infinite volume and then to the limit of a vanishing gluino mass. The results indicate that finite size effects are tolerable on lattices of moderate size, and that remaining deviations from supersymmetry are probably due to finite lattice spacing effects.

KEYWORDS: Supersymmetric gauge theory, Lattice QCD, Lattice Gauge Field Theories, Lattice Quantum Field Theory

ARXIV EPRINT: 1206.2341 


\section{Contents}

1 Introduction 1

2 Supersymmetric Yang-Mills theory on the lattice 3

3 Determination of masses 5

4 Finite size effects $\quad 8$

5 Conclusions 13

$\begin{array}{ll}\text { A Summary of the simulations and results } & 14\end{array}$

\section{Introduction}

Supersymmetric Yang-Mills theory (SYM) describes interacting gluons and their supersymmetric partners, the gluinos. As it is a gauge theory containing fermionic degrees of freedom, it is in this respect similar to QCD. An essential difference, however, is that gluinos are Majorana fermions in the adjoint representation of the gauge group. In Minkowski space the (on-shell) Lagrangian of SYM is composed out of the gluon fields $A_{\mu}$ and the gluino field $\lambda$, and reads

$$
\mathcal{L}=\operatorname{tr}\left[-\frac{1}{4} F_{\mu \nu} F^{\mu \nu}+\frac{\mathrm{i}}{2} \bar{\lambda} \gamma^{\mu} D_{\mu} \lambda-\frac{m_{g}}{2} \bar{\lambda} \lambda\right]
$$

where $F_{\mu \nu}$ is the usual non-Abelian field strength and $D_{\mu}$ denotes the gauge covariant derivative in the adjoint representation. The fields $\lambda$ and $A_{\mu}$ are transformed into each other by the supersymmetry transformation. The gluino mass term breaks supersymmetry softly.

Some properties of SYM are expected to be similar to QCD [1]. It is asymptotically free and is assumed to show confinement. The determination of its low-energy properties, including the spectrum of particles, is a non-perturbative problem. The "physical" particles are bound states of gluons and gluinos, and if supersymmetry is unbroken, they would form supermultiplets.

There are several motivations for the numerical investigation of SYM on the lattice. One of them is to understand the non-perturbative interaction of the gluinos in supersymmetric extensions of the standard model. There exist theoretical predictions for the low energy effective theory $[2,3]$ that can be compared to the lattice results. Another motivation for the numerical investigations of SYM on the lattice is related to possible connections to ordinary QCD, as provided by the orientifold planar equivalence [4]. Results of previous investigations of SYM on the lattice by our collaboration can be found in $[5,6]$.

In recent years, SYM on the lattice has also been investigated with Ginsparg-Wilson fermions, in the domain wall [7-9] as well as in the overlap formulation [10]. For large lattice 
volumes and small lattice spacings these formulations require, however, a significantly larger amount of computing resources than the Wilson formulation. The gain of no need for tuning the position of the zero gluino mass point does not compensate by far the advantage of Wilson fermions.

Our current studies of SYM are focussed on the bound states of gluons and gluinos, in particular on an exotic particle state in the spectrum that arises due to the fermions being in the adjoint representation. This particle is a spin 1/2 Majorana fermion and is called gluino-glue. It can be created by operators combining the field strength and the gluino field, the simplest example being

$$
\tilde{O}_{g \tilde{g}}=\sum_{\mu \nu} \sigma_{\mu \nu} \operatorname{tr}\left[F^{\mu \nu} \lambda\right]
$$

with $\sigma_{\mu \nu}=\frac{1}{2}\left[\gamma_{\mu}, \gamma_{\nu}\right]$. Such a bound state containing a single fermion does not occur in QCD, but analogous particles exist in models similar to QCD with an arbitrary number of quark flavours in the adjoint representation.

Since supersymmetry is generically broken in any non-trivial theory on the lattice [11] it has to be ensured that it is restored in the continuum limit. A necessary condition for unbroken supersymmetry is the degeneracy of fermionic and bosonic masses. In a supersymmetric theory the fermionic gluino-glue state must therefore be part of a multiplet containing also bosonic particles with the same mass. The behaviour of the gluino-glue particle provides an important signal for the supersymmetric limit of the theory.

It is expected that in SYM a fine-tuning of the bare gluino mass parameter in the continuum limit is enough to approach the symmetries of the continuum theory [12]. These symmetries include chiral symmetry and supersymmetry. The theoretical prediction of the existence of a supersymmetric chiral continuum limit needs to be confronted with the numerical lattice simulations. The chiral limit, the continuum limit, and the infinite volume limit can only be extrapolated from the results of these simulations. In QCD there are good estimates about the scales and parameters that are necessary to get reliable results, together with systematic estimates of the induced errors. In SYM the influence of parameters like the finite volume and non-zero lattice spacing may be different. It is therefore important to have a detailed understanding of their effects in order to be able to approach the supersymmetric limit.

The previous results of our simulations have not yet shown the expected degeneracy of the fermionic and bosonic masses [5, 6]. The obtained mass of the gluino-glue has been larger than the other masses of its lightest possible superpartners. However, the masses were obtained at a fixed lattice spacing and without a detailed analysis of the finite size effects. Our most recent investigations indicate that the influence of the finite lattice spacing is larger than expected. This provides a possible source of the supersymmetry breaking in the simulation.

In this work we want to scrutinize the influence of the finite volume. If it is large, it provides another possible explanation for the gap between fermionic and bosonic masses. If it is smaller than expected then the lattice artifacts have to be reduced by performing simulations at smaller lattice spacings. 
In our studies the mass of the gluino-glue particle has typically been the one determined with the best accuracy. Therefore it is well suited to estimate the effects of the finite lattice size. In order to estimate the influence on supersymmetry breaking, the mass of the adjoint version of the $\eta^{\prime}$ meson (a- $\eta^{\prime}$, a pseudoscalar bound state of gluinos) is also considered.

\section{Supersymmetric Yang-Mills theory on the lattice}

In our investigations we have employed the lattice formulation of SYM proposed by Curci and Veneziano [12]. The gauge field is represented by link variables $U_{\mu}(x)$ in the gauge group $\mathrm{SU}\left(N_{c}\right)$. The corresponding gauge action is the Wilson action built from the plaquette variables $U_{p}$. The gluinos are described by Wilson fermions in the adjoint representation. In its basic form the complete lattice action reads

$$
\mathcal{S}_{L}=\beta \sum_{p}\left(1-\frac{1}{N_{c}} \operatorname{Retr} U_{p}\right)+\frac{1}{2} \sum_{x y} \bar{\lambda}_{x}\left(\mathrm{D}_{\mathrm{w}}\right)_{x y} \lambda_{y},
$$

where $\mathrm{D}_{\mathrm{w}}$ is the Wilson-Dirac operator

$$
\begin{aligned}
\left(\mathrm{D}_{\mathrm{w}}\right)_{x, a, \alpha ; y, b, \beta}= & \delta_{x y} \delta_{a, b} \delta_{\alpha, \beta} \\
& -\kappa \sum_{\mu=1}^{4}\left[\left(1-\gamma_{\mu}\right)_{\alpha, \beta}\left(V_{\mu}(x)\right)_{a b} \delta_{x+\mu, y}+\left(1+\gamma_{\mu}\right)_{\alpha, \beta}\left(V_{\mu}^{\dagger}(x-\mu)\right)_{a b} \delta_{x-\mu, y}\right]
\end{aligned}
$$

The hopping parameter $\kappa$ is related to the bare gluino mass via $\kappa=1 /\left(2 m_{g}+8\right)$.

While the link variables $U_{\mu}(x)$ are in the fundamental representation of the gauge group, the variables $V_{\mu}(x)$ in the Wilson-Dirac operator are the corresponding elements in the adjoint representation. In our present investigations the gauge group is $\mathrm{SU}(2)$, the fundamental representation is a doublet and the adjoint one a triplet. In this case the adjoint gauge field is given by $\left[V_{\mu}(x)\right]^{a b}=2 \operatorname{tr}\left[U_{\mu}^{\dagger}(x) T^{a} U_{\mu}(x) T^{b}\right]$, where $T^{a}$ are the generators of the gauge group, normalised such that $2 \operatorname{tr}\left[T^{a} T^{b}\right]=\delta^{a b}$.

In our simulations the basic form of the lattice action has been modified with the following improvements: in order to reduce the lattice artifacts a tree-level Symanzik improved gauge action has been used instead of the simple Wilson gauge action. One level of stout smearing has been applied to the link fields in the Wilson-Dirac operator to reduce lattice artifacts also in the fermionic part of the action.

The lattice action explicitly breaks supersymmetry and in addition the chiral $\mathrm{U}(1)_{R}$ symmetry. However, to recover the continuum symmetries the necessary fine-tuning of supersymmetry and the $\mathrm{U}(1)_{R}$ symmetry can be achieved through the same parameter, the bare gluino mass (i.e. $\kappa$ ) [12]. The supersymmetric continuum limit coincides with the chiral continuum limit of the theory.

An important similarity of SYM to Yang-Mills theory is confinement. The static potential between quark sources in the fundamental representation of the gauge group shows no signal of a string breaking. The low energy effective action is built out of bound states of the elementary fields. The glueballs of Yang-Mills theory are completed with the mesonic states (gluino-balls) and the fermionic gluino-glue particle. The proposed supersymmetric low energy effective actions are constructed from two multiplets of particles $[2,3]$. The 
lighter multiplet consists of glueballs and a gluino-glue state. The heavier multiplet is built from the mesons and a gluino-glue state. As required by supersymmetry, each multiplet consists of a scalar, a pseudoscalar, and a fermionic spin $1 / 2$ particle.

On the lattice the $0^{++}$and the $0^{-+}$glueball operators are constructed from the link variables. The mesonic particles are the $\mathrm{a}-f_{0}$ represented by the operator $\bar{\lambda} \lambda$ and the $\mathrm{a}-\eta^{\prime}$ by $\bar{\lambda} \gamma_{5} \lambda$. Since the model contains only one species ("flavour") of gluinos, all possible mesons are "flavour diagonal". Consequently, their correlation functions contain disconnected parts in addition to the connected ones, as it is the case in QCD for flavour diagonal mesons.

In addition to the above mesons, we consider the adjoint pion $(\mathrm{a}-\pi)$, which is the pion in the corresponding theory with two Majorana fermions in the adjoint representation. The correlator of this particle is the connected contribution of the a- $\eta^{\prime}$ correlator. The $\mathrm{a}-\pi$ is not a physical particle in SYM, which only contains one Majorana fermion. However, it can be defined in a partially quenched setup, in which the model is supplemented by a second species of gluinos and the corresponding bosonic ghost gluinos, in the same way as for one-flavour QCD [13].

The reason for considering the $\mathrm{a}-\pi$ is the following. In the limit of a vanishing gluino mass, the bare parameter $\kappa$ has to be tuned to the critical value $\kappa_{c}$ (chiral limit) that corresponds to the chiral theory in the continuum limit. The value of $\kappa_{c}$ is most easily obtained from the dependence of the a- $\pi$-mass on $\kappa$. On the basis of arguments involving the OZI-approximation of SYM [2], the adjoint pion mass is expected to vanish for a massless gluino. The a- $\pi$ yields a more precise signal for the tuning than the supersymmetric Ward identities. However, in previous studies we have checked that both signals lead to a consistent value of $\kappa_{c}[5]$.

For both the $\mathrm{a}-f_{0}$ and $\mathrm{a}-\eta^{\prime}$ mesons the correlators contain a significant contribution from disconnected diagrams (see section 3), especially on larger lattices and small adjoint pion masses. As in comparable cases in lattice QCD, this contribution leads to a bad signal to noise ratio in the correlators. Using the techniques detailed in section 3 , the gluino-glue mass can be obtained with an accuracy significantly better compared to the other particles of the spectrum (apart from the unphysical adjoint pion).

In previous investigations the predicted multiplet structure of the particles has not been found in the mass spectrum [5]. The mass of the gluino-glue particle appeared to be heavier than the masses of the scalar and pseudoscalar states. A breaking of supersymmetry in the theory on the lattice is induced by the finite lattice spacing and the finite lattice extent. The former is an unavoidable consequence of the discretisation; the latter is due to the anti-periodic (thermal) boundary conditions implemented for the gluinos. It is important to have an estimate of these effects. If they had an opposite sign, they could compensate each other and suggest a wrong supersymmetric point. Alternatively, they could sum up to the total supersymmetry breaking and it would be necessary to investigate both of them in order to disentangle their effects.

In our numerical simulations we have applied a polynomial hybrid Monte Carlo (PHMC) algorithm. In a two step procedure, in the Metropolis step a better polynomial approximation is used than in the integrator of the molecular trajectory. The remaining error is compensated by a reweighting. Further details of the simulations algorithm can be found in $[5,14]$. 


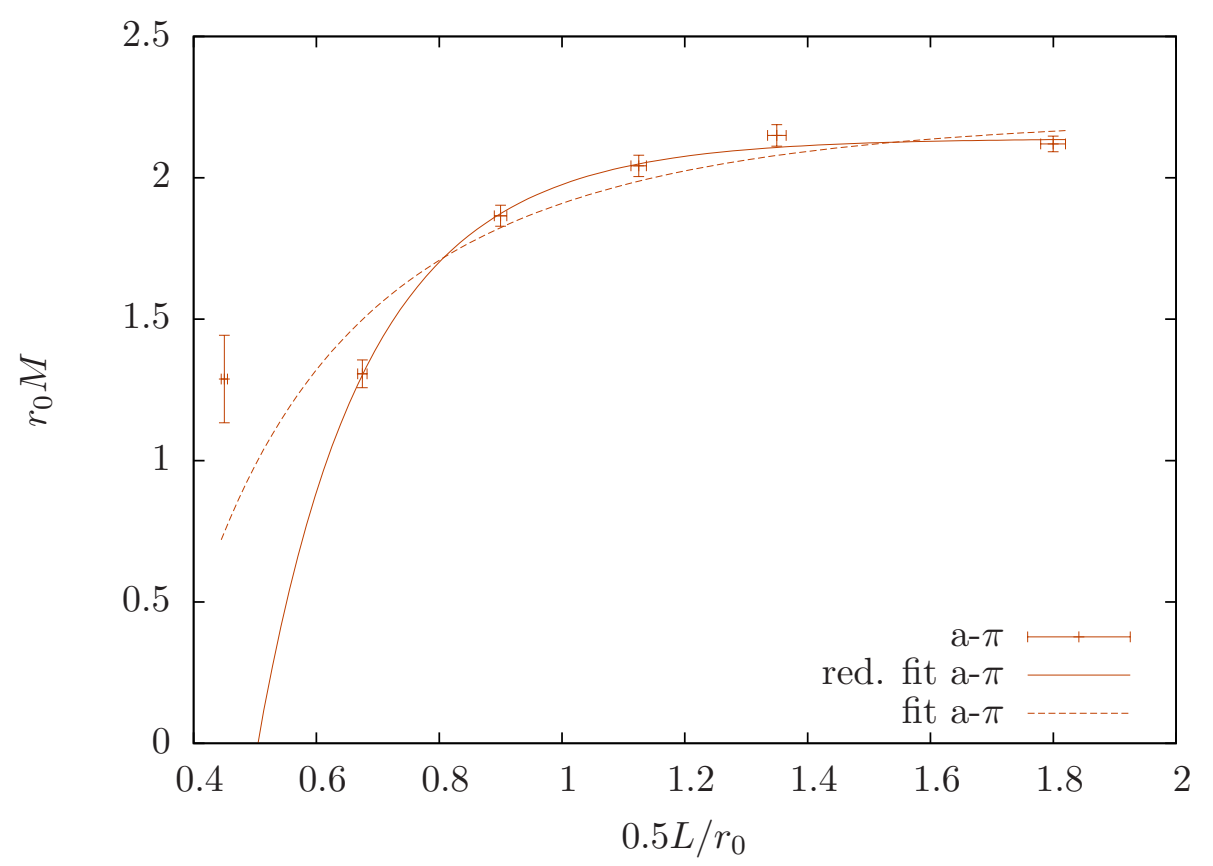

Figure 1. The mass of the adjoint pion $(a-\pi)$ at different lattice volumes for a fixed value of $\kappa=0.1490$. The fit (fit) is done using the ansatz eq. (4.2), and the reduced fit (red. fit) discards the smallest lattice volume. $r_{0}$ is the Sommer scale parameter.

The simulation at the smallest lattice spacings requires a large number of configurations to get a reasonable statistical error of the correlation functions. A further problem arises from the dependence of the adjoint pion mass on the lattice volume. This dependence is shown in figure 1 for a fixed value of $\kappa=0.1490$. The deviation of the smallest lattice from the infinite volume limit is most likely due to a larger influence of the excited states in the determination of the mass.

The adjoint pion mass gets smaller on the smaller lattices. Hence, for a fixed value of $\kappa$ the simulations are shifted towards the chiral limit when the lattice volume is reduced. In the vicinity of the chiral limit low eigenvalues of the Hermitian Wilson-Dirac operator occur. The polynomial approximation in the PHMC algorithm has a larger error in this case and a compensation with correction factors is necessary. To obtain these factors we have calculated an approximation of the lowest eigenvalue on each of the configurations. Whenever this value has been below the threshold determined by the polynomial approximation, the correct fermionic contribution of the 100 lowest eigenvalues has been calculated. From this contribution the correction factors of the reweighting step have been obtained. This reweighting leads to an additional increase of the statistical error on the smaller lattice volumes. A complete summary of the simulations is shown in table 3 .

\section{Determination of masses}

The operator for the gluino-glue particle is represented by the lattice version of eq. (1.2)

$$
O_{g \tilde{g}}^{\alpha}=\sum_{i<j, \beta} \sigma_{i j}^{\alpha \beta} \operatorname{tr}\left[P_{i j} \lambda^{\beta}\right]
$$


where the indices $i$ and $j$ stand for the spatial directions. In order to have the same properties with respect to parity transformation and time reversal, $F_{\mu \nu}$ is represented by the anti-Hermitian part of the clover plaquette $U^{(c)}[15]$,

$$
P_{i j}=\frac{1}{8 \mathrm{i} g_{0}}\left(U_{\mu \nu}^{(c)}-\left(U_{\mu \nu}^{(c)}\right)^{\dagger}\right) .
$$

The clover plaquette is a combination of links in the fundamental representation,

$$
\begin{aligned}
U_{\mu \nu}^{(c)}= & U_{\mu}(x) U_{\nu}(x+\mu) U_{\mu}^{\dagger}(x+\nu) U_{\nu}^{\dagger}(x) \\
& +U_{\nu}^{\dagger}(x-\nu) U_{\mu}(x-\nu) U_{\nu}(x-\nu+\mu) U_{\mu}^{\dagger}(x) \\
& +U_{\mu}^{\dagger}(x-\mu) U_{\nu}^{\dagger}(x-\mu-\nu) U_{\mu}(x-\mu-\nu) U_{\nu}(x-\nu) \\
& +U_{\nu}(x) U_{\mu}^{\dagger}(x+\nu-\mu) U_{\nu}^{\dagger}(x-\mu) U_{\mu}(x-\mu) .
\end{aligned}
$$

The best signal is obtained from the contribution to the correlator that is proportional to the identity in Dirac space. The corresponding correlator is

$$
C_{g \tilde{g}}\left(x_{0}-y_{0}\right)=-\frac{1}{4} \sum_{i, j, k, l} \sum_{\vec{x}, \vec{y}} \sum_{\alpha, \beta, \rho} \sum_{a b}\left\langle\sigma_{i j}^{\alpha \beta} \operatorname{tr}\left[P_{i j}(x) T^{a}\right]\left(\mathrm{D}_{\mathrm{w}}{ }^{-1}\right)_{x, a, \beta, y, b, \rho} \operatorname{tr}\left[P_{k l}(y) T^{b}\right] \sigma_{k l}^{\alpha \rho}\right\rangle .
$$

At large distances the correlator has the functional form

$$
C_{g \tilde{g}}(t) \approx C \sinh (m(t-T / 2)),
$$

where $T$ is the temporal extent of the lattice. The mass $m$ of the particle can be obtained by fitting the correlator to this function. The appropriate $t$-range for the fit, where $t$ is large enough but still much smaller than $T$, can be found by plotting the effective mass $m_{\mathrm{eff}}(t) . m_{\mathrm{eff}}(t)$ is the parameter $m$ obtained from the correlator at $t$ and $t+1$ assuming eq. (3.4) to be valid. In the $t$-range, where the influence of the excited states is small enough, $m_{\text {eff }}(t)$ shows a plateau. In a more refined approach the correlated chi-square of the fit provides an indication that the assumption of the eq. (3.4) is a good approximation in the considered region. We have applied a method that combines fit values of several fitting intervals and their correlated chi-square to get an estimation of the mass and the statistical and systematic error $[16,17]$.

The gluino-glue correlator has been obtained using different smearing techniques. The link fields are smeared using APE smearing, the fermionic fields using Jacobi smearing. Without any smearing the links $U$ in $P_{\mu \nu}$ should be the same fundamental links as in the gauge part of the action. The Wilson-Dirac operator $\mathrm{D}_{\mathrm{w}}$, on the other hand, should contain the one-level stout smeared links in the adjoint representation, that are used in the fermionic part of the action. In addition to this approach, the one-level stout smearing has also been applied to the $U$ fields. It has been checked that the smearing in the time-like direction included in this step has no influence on the correlation function at distances relevant for the mass determination.

The effect of the smearing on the effective masses is shown in figure 2. The same Jacobi smearing is applied on the source and sink side of the correlator. The APE and 


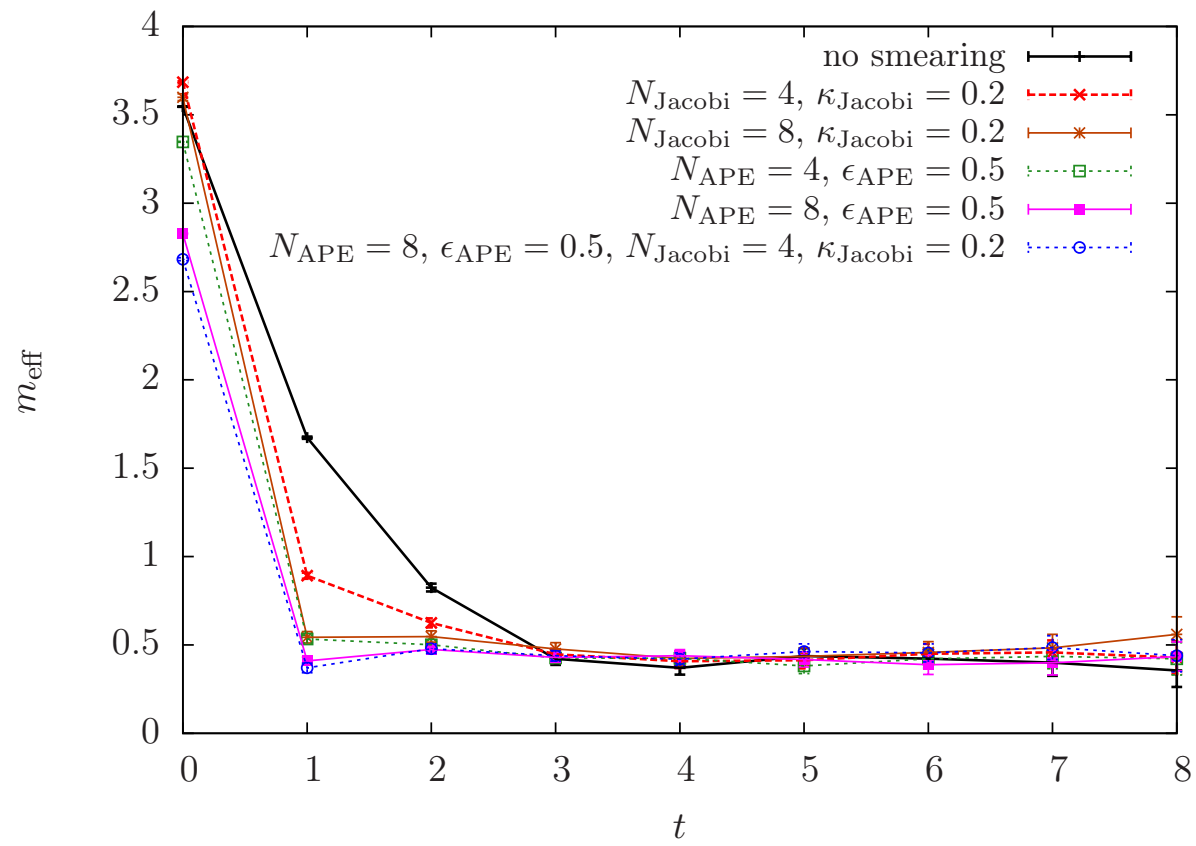

Figure 2. The effective mass of the gluino-glue on a $16^{3} \times 36$ lattice and $\kappa=0.1492$. The impact of different smearing parameters are compared. APE smearing is used on the the link fields in $P$, Jacobi smearing is applied on the fermionic source and sink.

the Jacobi smearing have a similar impact on the correlator. The excited states at small distances are considerably reduced by the smearing procedure.

The correlator for the $\mathrm{a}-\eta^{\prime}$ boson contains a connected and a disconnected contribution,

$$
\begin{aligned}
C_{\mathrm{a}-\eta^{\prime}} & =C_{\mathrm{a}-\pi}-C_{\mathrm{a}-\eta^{\prime}} \text { disc } \\
& =\frac{1}{L^{3}} \sum_{\vec{x}}\left\langle\operatorname{tr}\left[\gamma_{5}\left(\mathrm{D}_{\mathrm{w}}\right)_{x, y}^{-1} \gamma_{5}\left(\mathrm{D}_{\mathrm{w}}\right)_{y, x}^{-1}\right]\right\rangle-\frac{1}{2 L^{3}} \sum_{\vec{x}}\left\langle\operatorname{tr}\left[\gamma_{5}\left(\mathrm{D}_{\mathrm{w}}\right)_{x, x}^{-1}\right] \operatorname{tr}\left[\gamma_{5}\left(\mathrm{D}_{\mathrm{w}}\right)_{y, y}^{-1}\right]\right\rangle .
\end{aligned}
$$

Its connected part is the correlator of the adjoint pion $\left(C_{\mathrm{a}-\pi}\right)$. The disconnected part has been calculated using the stochastic estimator method [18]. The statistical fluctuations in this part are usually larger than in the connected contribution.

It is instructive to compare the masses of the $\mathrm{a}-\eta^{\prime}$ and the $\mathrm{a}-\pi$, since their difference shows the influence of the disconnected part. In figure 3 the dependence of these masses on the lattice volume is shown. On the smallest lattice both masses deviate from the behaviour expected from the fits. This is presumably due to the larger contribution of excited states to the correlator, since only smaller values of $t$ enter. A second observation is that at smaller lattice volumes both masses appear to approach each other. Thus at smaller volumes the influence of the disconnected part of the a $-\eta^{\prime}$ correlator is relatively smaller compared to the connected one.

At small $t$ the contribution of the connected part is much larger than the disconnected part, whereas at intermediate distances and close to the chiral limit the disconnected part yields a significant contribution. The larger statistical error of the $\mathrm{a}-\eta^{\prime}$ on the largest 


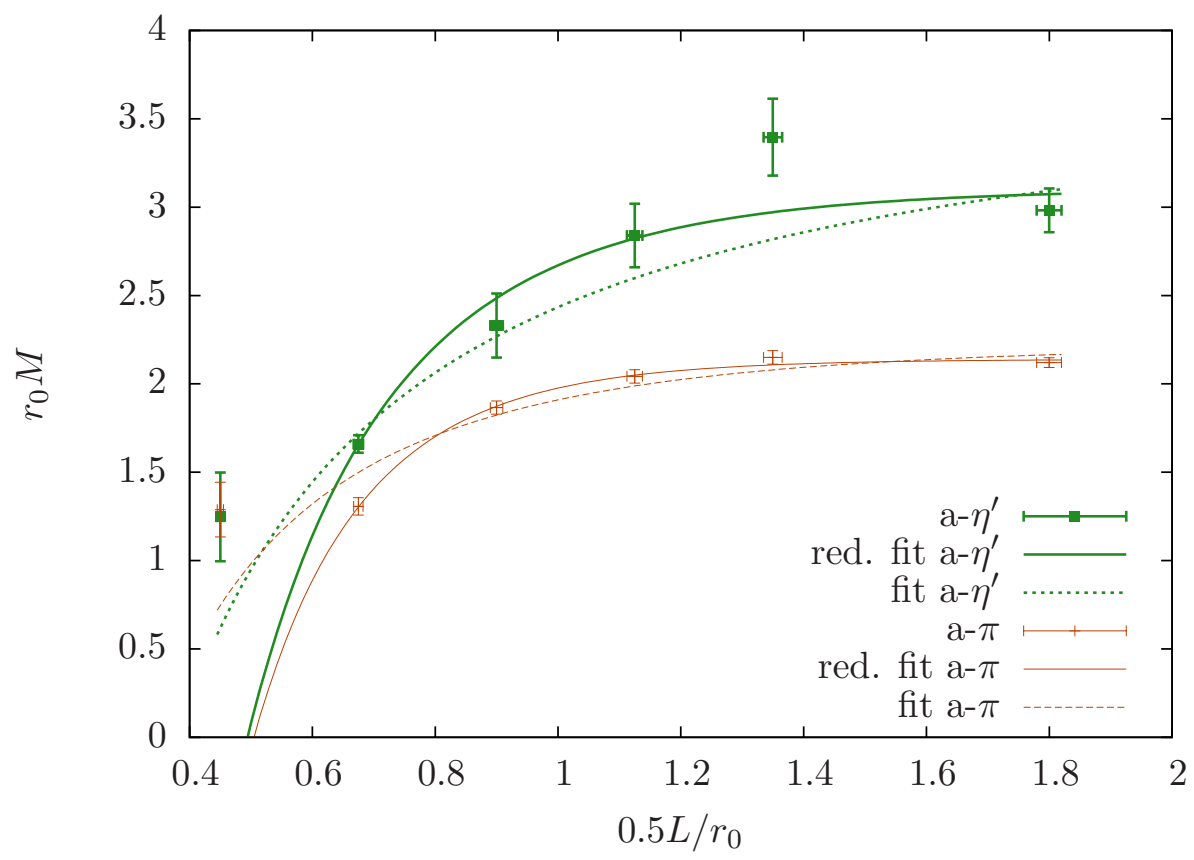

Figure 3. The masses of the $\mathrm{a}-\eta^{\prime}$ and the adjoint pion $(\mathrm{a}-\pi)$ at different lattice volumes for a fixed value of $\kappa=0.1490$. The fit (fit) is done using the ansatz eq. (4.2), and the reduced fit (red. fit) discards the smallest lattice volume.

lattices is due to the larger statistical fluctuations of the disconnected part. The total error on a small lattice seems to be underestimated for the a- $\eta^{\prime}$ particle due to these systematic uncertainties. Smearing techniques or a larger temporal extent of the lattice could lead to better results. Because of these systematic uncertainties of the a- $\eta^{\prime}$ mass and the larger statistical error, the best signal for the finite size effects is obtained from the gluino-glue mass.

\section{Finite size effects}

In quantum field theory in a finite volume the propagation and interactions of particles are different from those in an infinite volume. This can be understood as the modification of the polarized vacuum within a Compton wavelength around a particle due to the deformation by the finite boundaries of the box. The infinite volume mass $m_{0}$ of the particle is shifted due to the influence of the finite box size $L$,

$$
m(L)=m_{0}+\Delta m(L)
$$

The mass shift $\Delta m(L)$ has been studied to all orders in perturbation theory in massive field theories in the continuum in [19]. A similar investigation in the framework of field theory on the lattice has been made in [20]. The asymptotic behaviour was found to be

$$
\Delta m(L) \approx C L^{-1} \exp \left(-\alpha m_{0} L\right),
$$




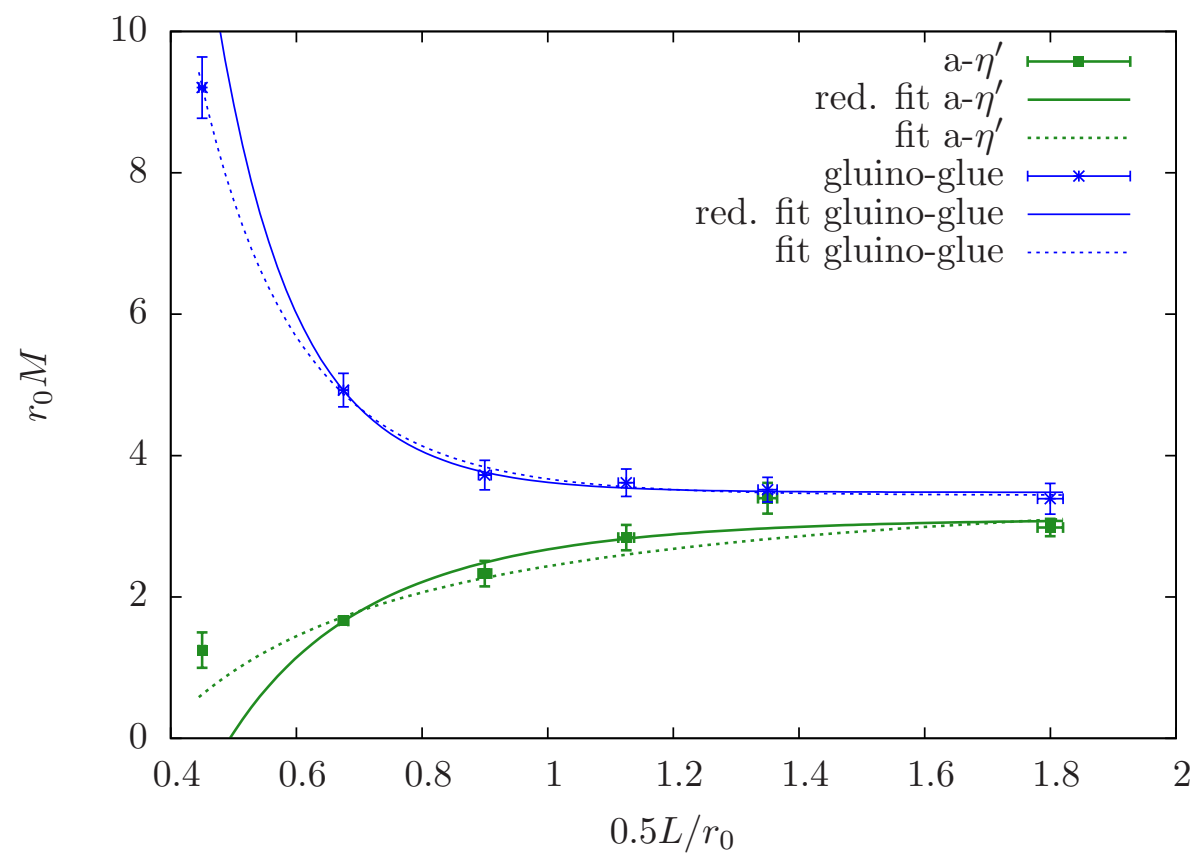

Figure 4. The masses of the gluino-glue and the a- $\eta^{\prime}$ meson at different lattice volumes for a fixed value of $\kappa=0.1490$. The fit (fit) is done using the ansatz eq. (4.2), and the reduced fit (red. fit) discards the smallest lattice volume.

with parameters $C$ and $1 \leq \alpha \leq \sqrt{3} / 2$. This behaviour is quite generic, since it does not depend on the specific form of the interactions. It also applies to the masses of stable bound states in a confining theory. For glueballs in lattice gauge theory the constants are [20]

$$
C=-\frac{3}{16 \pi} \frac{\lambda^{2}}{m_{0}^{2}}, \quad \alpha=\frac{\sqrt{3}}{2},
$$

where $\lambda$ is the three-glueball coupling constant. We fitted the dependence of the masses on the finite box size $L$ by the general asymptotic relation eq. (4.2) and obtained in this way an extrapolation to the infinite volume limit. To get more stable results, we have used a numerical fit to obtain the constants $m_{0}, C$, and $\alpha$.

We have carried out simulations at several box sizes $L$, see table 3 . The temporal extent $T$ of the lattice has been chosen to be about twice the spatial extent $L$. This combination has been chosen since it is the usual setup in all our simulations.

The first estimation of finite size effects is done at a fixed value of the bare gluino mass defined by the hopping parameter $\kappa=0.1490$. To visualize the influence on the mass gap between bosonic and fermionic states, the gluino-glue mass is shown in figure 4 together with the mass of the bosonic a- $\eta^{\prime}$ meson. Our ansatz eq. (4.2) for the functional dependence of the mass on the lattice volume is valid only in the asymptotic region of large $L$ and might fail for the smallest lattice sizes. To check its validity we have done a second fit (red. fit) that excludes the smallest lattice volume. The dimensionless scale $0.5 \mathrm{~L} / \mathrm{r}_{0}$ corresponds to the length in femtometers if the Sommer parameter $r_{0}$ is set to $r_{0}=0.5 \mathrm{fm}$ 


\begin{tabular}{|c|c|c|c|c|c|c|}
\hline$\left(r_{0} m_{\mathrm{a}-\pi}\right)^{2}$ & $m_{0 r}$ (fit) & $C$ (fit) & $\alpha$ (fit) & $m_{0 r}$ (red. fit) & $C$ (red. fit) & $\alpha$ (red. fit) \\
\hline 1 & $2.85(13)$ & $33.6(71)$ & $0.68(11)$ & $2.74(19)$ & $10.8(84)$ & $0.39(26)$ \\
2 & $3.010(81)$ & $30.0(43)$ & $0.627(65)$ & $2.94(11)$ & $12.4(72)$ & $0.41(17)$ \\
3 & $3.165(48)$ & $25.0(28)$ & $0.555(46)$ & $3.108(60)$ & $14.0(47)$ & $0.426(92)$ \\
4 & $3.362(28)$ & $22.7(16)$ & $0.516(26)$ & $3.345(41)$ & $18.9(52)$ & $0.481(66)$ \\
5 & $3.543(56)$ & $22.3(33)$ & $0.510(54)$ & $3.578(70)$ & $35(23)$ & $0.60(15)$ \\
\hline
\end{tabular}

Table 1. Fit parameters for the fits of the finite size effects shown in figure 6 .

as in QCD. Note that we have always taken the value of $r_{0}$ obtained by an extrapolation to the chiral limit of the data obtained on the $24^{3} \times 48$ and $32^{3} \times 64$ lattices.

The gluino-glue gets a positive mass shift at smaller volumes, whereas the a- $\eta^{\prime}$ gets a negative one. This clearly shows that the finite lattice size enhances the supersymmetry breaking induced by lattice discretisation and nonvanishing gluino mass in our model: at smaller lattices an additional splitting of the bosonic and fermionic masses is introduced.

In our previous simulations at larger box sizes the gluino-glue particle has been the heaviest of the measured low energy states. Since finite size effects are small in this case, this indicates that the mass shift induced by the discretisation artifacts is also positive. So, both finite size and finite lattice spacing effects add up to a total mass splitting between bosonic and fermionic states indicating the breaking of supersymmetry.

In case of the gluino-glue, eq. (4.2) seems to describe the volume dependence of the mass better than for the $\mathrm{a}-\eta^{\prime}$. Also, the fit for the a- $\eta^{\prime}$ fails for the smallest lattice volume. One possible reason for the different behaviour is the smearing of the gluino-glue that reduces the contribution of the excited states. This reduction becomes important for the smallest lattice sizes, where the masses are obtained from fits of the correlation function at smaller distances. Another reason are the systematic uncertainties in the mass determination of the $\mathrm{a}-\eta^{\prime}$ at smaller lattice sizes (see section 3). Nevertheless, neglecting the smallest lattice size, the fit seems to reproduce the general behaviour of the mass gap at different lattice volumes.

Above a box size of about $L=1.2 r_{0} / 0.5$ (corresponding to $1.2 \mathrm{fm}$ in QCD units) the statistical errors and the systematic errors of the finite size effects are of the same order and hence the finite size effects can be neglected at present. This is an important estimate for the minimal lattice size required for the simulations.

In the following we focus our discussion on the gluino-glue since the signal of the finite size effects is much clearer for this particle. Final results for masses in SYM should be obtained as extrapolations towards the infinite volume limit, the continuum limit, and the chiral limit. The continuum limit will be the subject of future investigations. The closer one gets to the chiral limit, the smaller are the masses of the particles and the ratio of the box size to the Compton wavelength. In other words, the finite size effects are expected to get larger in the vicinity of the chiral limit. Therefore, the proper procedure is an extrapolation to the infinite volume limit before the extrapolation of the chiral limit. 


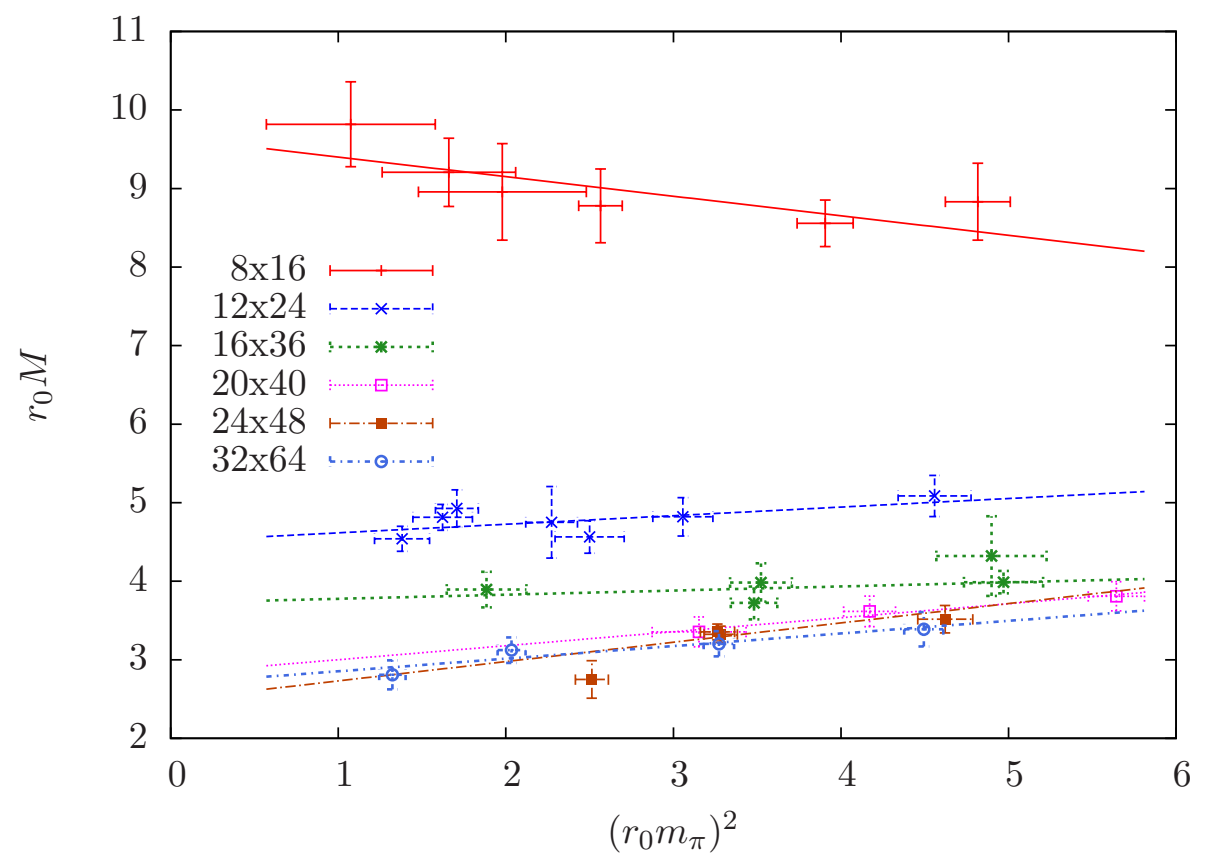

Figure 5. The mass of the gluino-glue at different lattice volumes and different values of $\kappa$ as a function of the square of the adjoint pion mass in units of the Sommer scale. The lines are obtained from a linear regression of the points for each lattice volume. They are used as a linear interpolation.
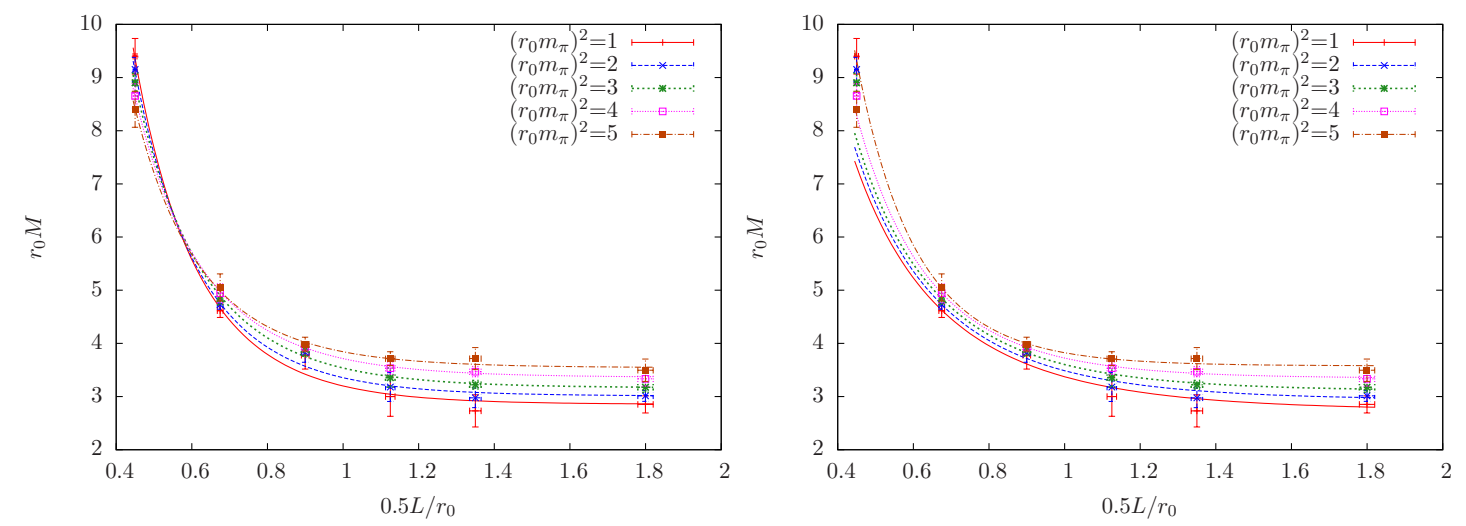

Figure 6. A fit of the finite size effects at several fixed values of $\left(r_{0} m_{\mathrm{a}-\pi}\right)^{2}$. The fit is done using the ansatz eq. (4.2). The right panel shows the fits neglecting the smallest lattice volume.

In the first step we have extrapolated the values of the masses to the infinite volume limit but away from the chiral limit. Instead of a fixed value of the bare parameter $\kappa$, we have fixed the squared mass of the adjoint pion, $\left(r_{0} m_{\mathrm{a}-\pi}\right)^{2}$, to five different values. The masses of the gluino-glue at these values have been obtained from a linear interpolation of the simulation results at each box size. These interpolations are shown in figure 5. The masses at the three largest box sizes are almost indistinguishable in view of the current statistical errors. 


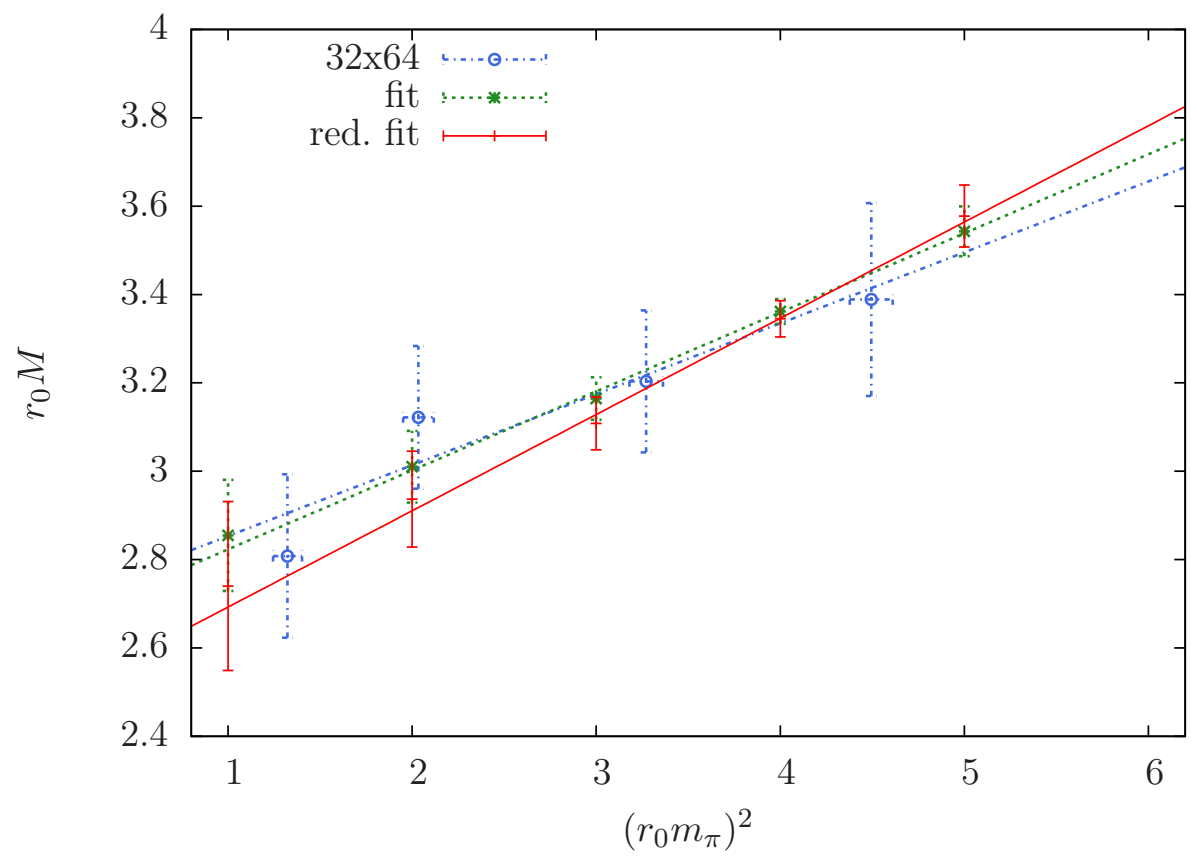

Figure 7. The gluino-glue mass extrapolated to the infinite volume limit as a function of the squared mass of the adjoint pion in units of the Sommer scale. In addition the mass is shown at the largest lattice volume and different values of $\kappa$.

\begin{tabular}{|c|c|c|c|c|c|c|c|}
\hline $8 \times 16$ & $12 \times 24$ & $16 \times 36$ & $20 \times 40$ & $24 \times 48$ & $32 \times 64$ & fit & red. fit \\
\hline $9.65(46)$ & $4.51(20)$ & $3.72(34)$ & $2.82(47)$ & $2.48(42)$ & $2.69(23)$ & $2.644(91)$ & $2.47(12)$ \\
\hline
\end{tabular}

Table 2. The lower entries of this table are the results of chiral extrapolations of the gluino-glue mass at fixed lattice sizes. The last two entries are obtained by first performing the extrapolation to the infinite volume limit and then to the chiral limit. In the reduced fit (red. fit) the smallest lattice volume is ignored. All masses are in units of the Sommer scale.

The results of the interpolation are shown in figure 6 . The lines correspond to the fit of the dependence on the finite box size according to eq. (4.2). The parameter $m_{0}$ of the fit is the extrapolated infinite volume limit of the masses. The different parameters of the fits are summarised in table 1.

The masses extrapolated to the infinite volume are shown in figure 7 as a function of $\left(r_{0} m_{\mathrm{a}-\pi}\right)^{2}$. For comparison, the results of the largest lattice have been added to this plot.

From the masses at different values of $\left(r_{0} m_{\mathrm{a}-\pi}\right)^{2}$ the chiral extrapolation can be obtained assuming a linear dependence. The linear regression of this dependence can be seen in figure 7. The values obtained from linear extrapolations to the chiral limit are displayed in table 2. The table contains the values at fixed lattice sizes as well as the results from the infinite volume extrapolations. The results at the largest two lattices appear to be consistent with those from the infinite volume limit. 


\section{Conclusions}

In this work we have investigated the implications of the finite lattice extent in the simulations of SYM on a lattice. An exotic particle of SYM, the gluino-glue, turned out to provide the best signal for the finite volume dependence of the masses. A comparison with the bosonic a- $\eta^{\prime}$ meson shows that the finite size effects on the masses have opposite signs for both particles: the gluino-glue gets a larger mass at smaller box size, whereas the mass of the $\mathrm{a}-\eta^{\prime}$ is reduced. This indicates that finite size effects enhance the supersymmetry breaking induced by lattice discretisation and nonvanishing gluino mass, and increase the unexpected gap between the bosonic and fermionic states seen in previous lattice results. The influence of the finite box size decreases, however, quite rapidly. The differences between the three largest lattices in our simulations are nearly negligible. The physical reason for this effect is the large mass of the lightest particle in this theory. The adjoint pion, which is light and becomes massless in the chiral limit, is not a physical particle and is only defined in a partially quenched framework. The lightest physical particles have significantly larger masses, even in the chiral limit. Therefore their Compton wavelengths are small compared to the sizes of our largest lattices.

The aim of these investigations was to obtain estimates of the finite size effects and their implications on the setup for future simulations of SYM. Another item would be to consider the effect of changing the fermionic temporal boundary conditions from antiperiodic to periodic. This could help to disentangle the effects of the finite spatial volume and the finite temporal extent. In our present work we have chosen a ration of $L$ over $T$ similar to our final simulations for the determination of the complete spectrum on larger lattices.

The findings have implications for our further investigations of SYM. We have found that finite size effects are small for $L \geq 1.2 r_{0} / 0.5$ (corresponding to $1.2 \mathrm{fm}$ in QCD units), and that the simulations can be efficiently done at present lattice spacings on medium lattice sizes, for instance, $24^{3} \times 48$. In order to obtain reliable results relevant to the continuum limit a sufficiently large statistics and a small lattice spacing appear to be crucial.

\section{Acknowledgments}

This project is supported by the German Science Foundation (DFG) under contract $\mathrm{Mu}$ $757 / 16$, and by the John von Neumann Institute for Computing (NIC) with grants of computing time. Further computing time has been provided by the compute cluster PALMA of the University of Münster. 


\section{A Summary of the simulations and results}

\begin{tabular}{|c|c|c|c|c|c|c|c|c|}
\hline$L \times T$ & $0.5 L / r_{0}$ & $\kappa$ & $a m_{\mathrm{a}-\pi}$ & $\left(r_{0} m_{\mathrm{a}-\pi}\right)^{2}$ & $a m_{g \tilde{g}}$ & $a m_{\mathrm{a}-\eta^{\prime}}$ & $N_{\text {conf }}$ & $N_{\text {corr }}$ \\
\hline $8 \times 16$ & $0.4499(51)$ & 0.1475 & $0.2469(22)$ & $4.82(19)$ & $0.993(44)$ & $0.2388(29)$ & 12573 & - \\
\hline $8 \times 16$ & $0.4499(51)$ & 0.1478 & $0.2223(23)$ & $3.91(17)$ & $0.963(23)$ & $0.2140(30)$ & 12890 & 2 \\
\hline $8 \times 16$ & $0.4499(51)$ & 0.1482 & $0.1802(25)$ & $2.56(13)$ & $0.988(42)$ & $0.1760(41)$ & 5062 & 3 \\
\hline $8 \times 16$ & $0.4499(51)$ & 0.1487 & $0.158(18)$ & $1.98(50)$ & $1.008(58)$ & $0.159(16)$ & 9900 & 2 \\
\hline $8 \times 16$ & $0.4499(51)$ & 0.1490 & $0.145(16)$ & $1.66(40)$ & $1.036(37)$ & $0.140(27)$ & 59789 & 47 \\
\hline $8 \times 16$ & $0.4499(51)$ & 0.1491 & $0.117(26)$ & $1.07(50)$ & $1.104(48)$ & $0.108(20)$ & 59972 & 40 \\
\hline $12 \times 24$ & $0.6749(76)$ & 0.1485 & $0.2402(30)$ & $4.56(22)$ & $0.572(23)$ & $0.233(13)$ & 9316 & 1 \\
\hline $12 \times 24$ & $0.6749(76)$ & 0.1487 & $0.1967(36)$ & $3.06(18)$ & $0.542(21)$ & $0.2050(43)$ & 9096 & 11 \\
\hline $12 \times 24$ & $0.6749(76)$ & 0.1488 & $0.1778(53)$ & $2.50(21)$ & $0.513(18)$ & $0.1711(74)$ & 8774 & 16 \\
\hline $12 \times 24$ & $0.6749(76)$ & 0.1489 & $0.1696(39)$ & $2.27(15)$ & $0.534(45)$ & $0.1636(49)$ & 8073 & 20 \\
\hline $12 \times 24$ & $0.6749(76)$ & 0.1490 & $0.1470(39)$ & $1.71(13)$ & $0.554(21)$ & $0.1867(35)$ & 8083 & 225 \\
\hline $12 \times 24$ & $0.6749(76)$ & 0.1491 & $0.1433(62)$ & 1.62 & $0.541(12)$ & $0.1604(87)$ & 8238 & 158 \\
\hline $12 \times 24$ & $0.6749(76)$ & 0.1492 & $0.1322(64)$ & $1.38(16)$ & $0.511(12)$ & $0.147(12)$ & 8033 & 173 \\
\hline 36 & $0.900(10)$ & 0.1487 & $0.2508(31)$ & $4.97(24)$ & $0.449(11)$ & $0.349(22)$ & 4699 & - \\
\hline $16 \times 36$ & $0.900(10)$ & 0.1488 & $0.2490(56)$ & $4.90(33)$ & $0.486(52)$ & $0.281(14)$ & 1101 & 9 \\
\hline $16 \times 36$ & $0.900(10)$ & 0.1489 & $0.2111(31)$ & $3.52(18)$ & $0.448(22)$ & $0.228(32)$ & 4796 & 10 \\
\hline $16 \times 36$ & $0.900(10)$ & 0.1490 & 0.2099(18) & $3.48(14)$ & $0.419(19)$ & $0.262(17)$ & 9909 & 24 \\
\hline $16 \times 36$ & $0.900(10)$ & 0.1492 & $0.1544(80)$ & $1.89(24)$ & $0.438(21)$ & $0.263(17)$ & 7550 & 177 \\
\hline $20 \times 40$ & $1.125(13)$ & 0.1488 & $0.26723(96)$ & $5.64(17)$ & $0.428(16)$ & $0.323(19)$ & 3700 & - \\
\hline $20 \times 40$ & $1.125(13)$ & 0.1490 & $0.2297(17)$ & $4.17(15)$ & $0.407(17)$ & $0.319(17)$ & 3698 & 5 \\
\hline $20 \times 40$ & $1.125(13)$ & 0.1492 & $0.1997(66)$ & $3.15(28)$ & $0.377(17)$ & $0.37(11)$ & 3799 & 72 \\
\hline $24 \times 48$ & $1.350(15)$ & 0.1490 & $0.2418(16)$ & $4.62(16)$ & $0.396(15)$ & $0.382(20)$ & 6899 & 11 \\
\hline $24 \times 48$ & $1.350(15)$ & 0.1492 & $0.20321(89)$ & $3.26(10)$ & $0.3773(71)$ & $0.301(14)$ & 3228 & 81 \\
\hline $24 \times 48$ & $1.350(15)$ & 0.1492 & $0.20381(80)$ & $3.283(99)$ & $0.3740(75)$ & $0.299(28)$ & 8869 & - \\
\hline $24 \times 48$ & $1.350(15)$ & 0.1493 & $0.1783(15)$ & 2.513(98) & $0.309(23)$ & $0.289(19)$ & 10203 & 18 \\
\hline $32 \times 64$ & $1.800(20)$ & $\mid 0.1490$ & $0.23847(41)$ & $4.49(12)$ & $0.381(20)$ & $0.335(10)$ & 5039 & - \\
\hline $32 \times 64$ & $1.800(20)$ & 0.1492 & $0.20346(54)$ & $3.272(91)$ & $0.360(14)$ & $0.292(13)$ & 6348 & - \\
\hline $32 \times 64$ & $1.800(20)$ & 0.1494 & $0.1604(15)$ & $2.034(83)$ & $0.351(14)$ & $0.269(25)$ & 5485 & 156 \\
\hline $32 \times 64$ & $1.800(20)$ & 0.1495 & $0.1294(24)$ & $1.323(79)$ & $0.316(17)$ & $0.253(45)$ & 2147 & 80 \\
\hline
\end{tabular}

Table 3. Summary of our simulations at $\beta=1.75$. The value of $r_{0} / a$ obtained from the extrapolation of the $24^{3} \times 48$ and $32^{3} \times 64$ lattice data to the chiral limit is $r_{0} / a=8.89(10)$. $N_{\text {conf }}$ is the number of configurations in the gluino-glue mass measurement. $N_{\text {corr }}$ is the total number of configurations with a reweighting factor different from one.

Open Access. This article is distributed under the terms of the Creative Commons Attribution License which permits any use, distribution and reproduction in any medium, provided the original author(s) and source are credited. 


\section{References}

[1] D. Amati, K. Konishi, Y. Meurice, G. Rossi and G. Veneziano, Nonperturbative aspects in supersymmetric gauge theories, Phys. Rept. 162 (1988) 169 [INSPIRE].

[2] G. Veneziano and S. Yankielowicz, An effective lagrangian for the pure $N=1$ supersymmetric Yang-Mills theory, Phys. Lett. B 113 (1982) 231 [INSPIRE].

[3] G. Farrar, G. Gabadadze and M. Schwetz, On the effective action of $N=1$ supersymmetric Yang-Mills theory, Phys. Rev. D 58 (1998) 015009 [hep-th/9711166] [INSPIRE].

[4] A. Armoni, M. Shifman and G. Veneziano, From super Yang-Mills theory to QCD: Planar equivalence and its implications, hep-th/0403071 [INSPIRE].

[5] K. Demmouche et al., Simulation of $4 D N=1$ supersymmetric Yang-Mills theory with Symanzik improved gauge action and stout smearing, Eur. Phys. J. C 69 (2010) 147 [arXiv: 1003.2073] [INSPIRE].

[6] G. Bergner, I. Montvay, G. Münster, D. Sandbrink and U.D. Özugurel, Supersymmetric Yang-Mills theory: a step towards the continuum, arXiv:1111.3012 [INSPIRE].

[7] G.T. Fleming, J.B. Kogut and P.M. Vranas, Super Yang-Mills on the lattice with domain wall fermions, Phys. Rev. D 64 (2001) 034510 [hep-lat/0008009] [INSPIRE].

[8] J. Giedt, R. Brower, S. Catterall, G.T. Fleming and P. Vranas, Lattice super-Yang-Mills using domain wall fermions in the chiral limit, Phys. Rev. D 79 (2009) 025015 [arXiv:0810.5746] [INSPIRE].

[9] M.G. Endres, Dynamical simulation of $N=1$ supersymmetric Yang-Mills theory with domain wall fermions, Phys. Rev. D 79 (2009) 094503 [arXiv:0902.4267] [INSPIRE].

[10] JLQCD collaboration, S. Kim et al., Lattice study of $4 D \mathcal{N}=1$ super Yang-Mills theory with dynamical overlap gluino, PoS(LATTICE2011)069 [arXiv:1111.2180] [INSPIRE].

[11] G. Bergner, Complete supersymmetry on the lattice and a no-go theorem, JHEP 01 (2010) 024 [arXiv:0909.4791] [INSPIRE].

[12] G. Curci and G. Veneziano, Supersymmetry and the lattice: a reconciliation?, Nucl. Phys. B 292 (1987) 555 [InSPIRE].

[13] F. Farchioni et al., Hadron masses in QCD with one quark flavour, Eur. Phys. J. C 52 (2007) 305 [arXiv:0706.1131] [INSPIRE].

[14] I. Montvay and E. Scholz, Updating algorithms with multi-step stochastic correction, Phys. Lett. B 623 (2005) 73 [hep-lat/0506006] [INSPIRE].

[15] A. Donini, M. Guagnelli, P. Hernández and A. Vladikas, Towards $N=1$ super Yang-Mills on the lattice, Nucl. Phys. B 523 (1998) 529 [hep-lat/9710065] [InSPIRE].

[16] European Twisted Mass collaboration, R. Baron et al., Computing $K$ and $D$ meson masses with $N_{f}=2+1+1$ twisted mass lattice QCD,

Comput. Phys. Commun. 182 (2011) 299 [arXiv: 1005.2042] [InSPIRE].

[17] I. Montvay and C. Urbach, Exploratory investigation of nucleon-nucleon interactions using Euclidean Monte Carlo simulations, Eur. Phys. J. A 48 (2012) 38 [arXiv:1105.5009] [INSPIRE].

[18] G.S. Bali, S. Collins and A. Schäfer, Effective noise reduction techniques for disconnected loops in lattice QCD, Comput. Phys. Commun. 181 (2010) 1570 [arXiv:0910.3970] [INSPIRE]. 
[19] M. Lüscher, Volume dependence of the energy spectrum in massive quantum field theories. 1. Stable particle states, Commun. Math. Phys. 104 (1986) 177 [INSPIRE].

[20] G. Münster, The size of finite size effects in lattice gauge theories, Nucl. Phys. B 249 (1985) 659 [inSPIRE]. 